\title{
Silicon Waveguide-coupled Octagonal Microresonators with Directional Coupling to Single Modes
}

\author{
Chao Li and Andrew W. Poon \\ Department of Electrical and Electronic Engineering, The Hong Kong University of Science and Technology, \\ Clear Water Bay, Hong Kong SAR, China \\ Tel: (852)-2358-7905, fax: (852)-2358-1485,email: eeawpoon@ust.hk
}

\begin{abstract}
We propose silicon waveguide-coupled octagonal microresonators with directional coupling to single modes. Numerical simulations suggest that the k-vectors in waveguide-coupled large-sized octagonal microstructures are directional. Experiments demonstrate two modes from a waveguide-coupled 50- $\mu \mathrm{m}$-size octagonal microresonator.

(C) 2006 Optical Society of America

OCIS codes: (230.5750) Resonators; (230.3990) Microstructure devices
\end{abstract}

High-Q optical microresonators in thin silicon-on-isolator (SOI) have received considerable attention as promising technologies for realizing photonic integrated circuits on silicon chips. Most of these works employ conventional microring [1] or circular microdisk [2] resonators. However, circular microring and microdisk have undesirable micrometer-scale curved sidewalls that impose a limited interaction length for lateral coupling with straight waveguides. Besides, waveguide-coupled microdisk resonators tend to be multimode. Previously, we proposed and demonstrated laterally waveguide-coupled polygonal microresonators in silicon nitride as an alternative microresonator-based device design [3]. The key merit of the polygonal microresonators is that the flat microcavity sidewalls enable long interaction lengths with laterally coupled straight waveguides. We also showed that polygonal microresonators with round cavity corners have enhanced Q factors. Here we report our analysis on waveguide-coupled round-cornered octagonal microresonators assuming an oxide-clad SOI substrates, and initial experimental demonstrations on a $50-\mu \mathrm{m}$-size round-cornered octagonal microresonator in thin-SOI substrates. Our numerical simulations and Fourier analysis suggest that waveguide lateral coupling to an octagonal microcavity flat sidewall that is sufficiently long enables directional coupling to single modes.

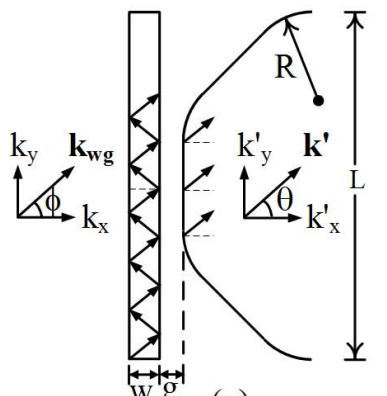

(a)

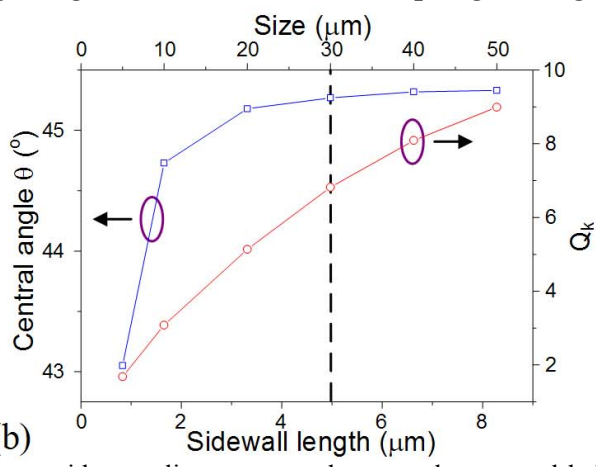

(b)

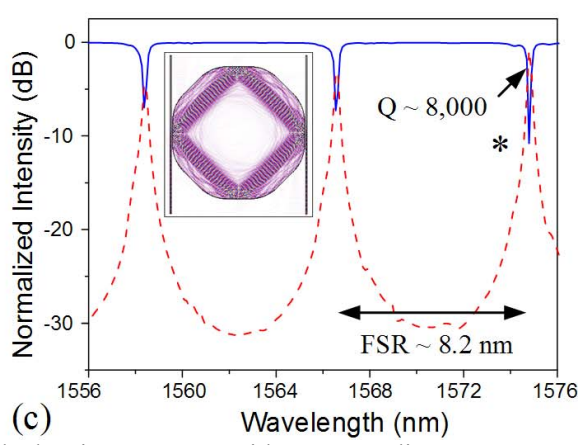

(c) Wavelength $(\mathrm{nm})$

and sidewall-to-sidewall distance L. $\mathbf{k}_{\mathrm{wg}}$ : waveguide mode $\mathbf{k}$-vector, $\phi: \mathbf{k}_{\mathrm{wg}}$ angle relative to the waveguide sidewall normal, k': coupled k-vector, $\theta$ : k' angle relative to the microcavity sidewall normal, w: waveguide width, g: gap spacing. (b) Simulated $\theta$ 's (squares) and $\mathrm{Q}_{\mathrm{k}}$ 's (circles) with various L's and sidewall lengths. (c) Simulated TE-polarized throughput(solid line) and drop-port (dashed line) spectra of a waveguide-coupled round-cornered octagonal microresonator add-drop filter. Inset shows the traveling-wave 4-bounce orbit field pattern at resonance “*”. The calculations assume $\mathrm{R} / \mathrm{L}=0.3$, w $=0.27 \mu \mathrm{m}$, silicon device refractive index $=3.5$, silica background (upper-cladding) refractive index $=1.44$, and $\mathrm{g}=0.3 \mu \mathrm{m}$.

Fig. 1(a) shows the schematics of waveguide coupling to a round-cornered octagonal microresonator. When a singlemode straight waveguide is evanescently input-coupled to a microstructure with a sufficiently long flat 
sidewall of the same refractive index $\mathrm{n}$, it is conceivable that the coupled $\mathbf{k}$-vectors can have angle $\theta$ 's centered about the waveguide mode $\mathbf{k}$-vector angle $\phi$ (relative to the waveguide sidewall normal). In contrast, the coupled $\mathbf{k}$-vectors along a relatively short arc length of a curved sidewall can have a relatively large angular distribution.

In order to reveal the coupled $\mathbf{k}$-vector distributions, we employ two-dimensional finite-difference time-domain (FDTD) simulations tool to simulate planar waveguide coupling to half-block round-cornered octagonal microstructures and microresonators. We then analyze the simulated field patterns in $\mathbf{k}$-space by means of Fourier transform. In order to quantify the coupled $\mathbf{k}$-vectors directionality, we define a quality factor $\mathrm{Q}_{\mathrm{k}}=\theta / \Delta \theta$. Fig. (b) shows the simulated $\theta$ 's and $\mathrm{Q}_{\mathrm{k}}$ 's of waveguide-coupled half-block round-cornered $(\mathrm{R} / \mathrm{L}=0.3)$ octagonal microstructures with various L's and sidewall lengths. We see that $\theta$ rises sharply with sidewall length and saturates at about $45.3^{\circ}$ as sidewall length exceeds about $5 \mu \mathrm{m}$, whereas $\mathrm{Q}_{\mathrm{k}}$ linearly increases with sidewall length. Thus, the coupled k-vectors become directional only with sufficiently-long flat coupling sidewalls. Fig. 1(c) shows the simulated throughput- and drop-port spectra of a waveguide-coupled round-cornered $(\mathrm{R} / \mathrm{L}=0.3)$ octagonal microresonator add-drop filter. In order to directionally couple to 4-bounce orbit modes, we choose $L=30 \mu \mathrm{m}$ (sidewall length $\sim 5 \mu \mathrm{m}$ ) based on Fig. 1(b). We observe single modes with a maximum extinction ratio of $\sim 11 \mathrm{~dB}$ and a $\mathrm{Q}$ of $\sim 8,000$. Inset shows the traveling-wave 4-bounce orbit mode field pattern at resonance "*". We remark that as the number of modes is determined by the coupled $\mathbf{k}$-vector distribution and the cavity mode density, only properly shaped and sized microresonators will perform singlemode.

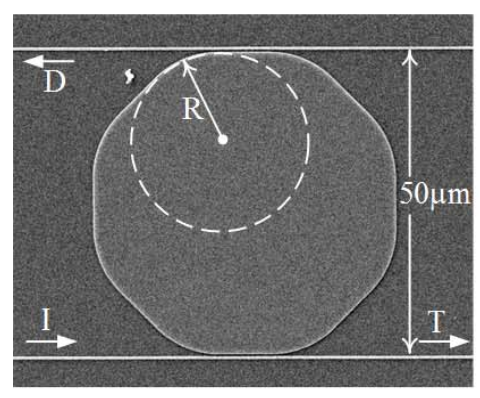

(a)

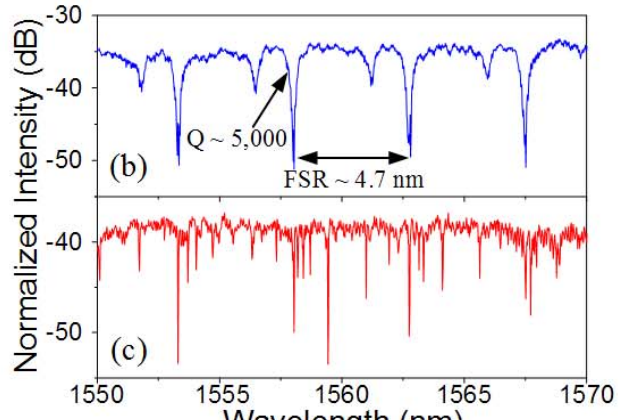

Wavelength $(\mathrm{nm})$

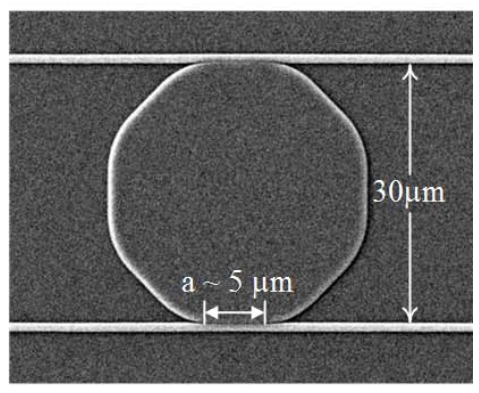

(d)

Fig.2. (a) Top-view scanning electron micrograph (SEM) of the waveguide-coupled round-cornered octagonal microresonator in thin SOI with an air upper-cladding. $\mathrm{R} / \mathrm{L}=0.3$. (b), (c) Measured TE-polarized throughput spectra of the waveguide-coupled round-cornered octagonal microresonator and a control circular microdisk resonator of $50 \mu \mathrm{m}$ size (d) Top-view SEM of the fabricated round-cornered octagonal microresonator in thin SOI with oxide cladding.

Fig. 2(a) shows the top-view scanning electron micrograph of our initially fabricated waveguide-coupled $50-\mu \mathrm{m}$-size octagonal $(\mathrm{R} / \mathrm{L}=0.3)$ microresonator in thin SOI with air upper-cladding. Fig. $2(\mathrm{~b})$ shows the measured TE-polarized throughput spectrum exhibiting only two distinct modes with a maximum extinction ratio of $\sim 18 \mathrm{~dB}$ and $\mathrm{Q} \sim 5,000$. The FSR is $\sim 4.7 \mathrm{~nm}$, which is consistent with the calculated FSR of $\sim 4.9 \mathrm{~nm}$ by assuming 4-bounce ray orbits. Fig. 2(c) shows the measured TE-polarized throughput spectrum of a waveguide-coupled $50-\mu \mathrm{m}$-size circular microdisk as a control. The spectrum displays highly multimode. Fig. 2(d) shows the top-view scanning electron micrograph of the fabricated $30-\mu \mathrm{m}$-size waveguide-coupled round-cornered octagonal microresonators with silica upper-cladding based on our analysis (Fig. 2). Device characterization and further device parameters optimization are in progress.

[1] T. Baehr-Jones, M. Hochberg, C. Walker, and A. Scherer, "High-Q ring resonators in thin silicon-on-insulator," Appl. Phys. Lett., 85, 3346-3347 (2004).

[2] T. Indukuri, P. Koonath, and B. Jalali, "Subterranean silicon photonics: demonstration of buried waveguide-coupled microresonators," Appl. Phys. Lett., 87, 081114 (2005).

[3] C. Li and A. W. Poon, "Experimental demonstration of waveguide-coupled round-cornered octagonal microresonators in silicon nitride", Opt. Lett., 30, 546-548 (2005). 